\title{
The Aging-related Approach to Detection of Mitotic Factor in Thoracic Duct Lymph of Rabbits
}

\author{
Alexander V. Kuznetsov ${ }^{1 \star}$, Ydyrys A. Almabayev ${ }^{2}$, Ildar R. Fackhradiev ${ }^{3}$, Aigul Y. Almabaeva ${ }^{4}$, \\ Jandos J. Serikpayev ${ }^{5}$, Baimakhan D. Tanabayev ${ }^{6}$
}

${ }^{1}$ Department of Human Anatomy, Novosibirsk State Medical University, Russia, ${ }^{2}$ Department of the Clinical Anatomy, Kazakh National Medical University, Kazakhstan, ${ }^{3}$ Laboratory of Experimental Medicine, Kazakh National Medical University, Kazakhstan, ${ }^{4,5}$ Department of Human Anatomy, O.S. Medical University Astana, Kazakhstan, ${ }^{6}$ Department of Morphological, South Kazakhstan State Pharmaceutical Academy, Kazakhstan

\section{ABSTRACT}

Introduction: It is known that mitosis is a fundamental process and it has been researched in normal as well as in pathology. It is suggested that the cause of a colossal number of research about mitosis is an empirical approach to the problem. For example, finding the optimal combination (MF) of only nine components (cytokines or any proteins) requires 181440 steps.

Aim: In the study, the aging-related approach to detection of an explicit of mitotic factor MF to control mitosis through only one step is offered.

Material and Methods: The following formula of MF has been invented: $\mathrm{MF}=\Delta(\mathrm{A}-\mathrm{B})$. The formula includes: (a) Composition of the known components of thoracic duct lymph (TDL) of immature rabbits and (b) composition of the known components of mature rabbits TDL, $\Delta$ - the difference of the components composition between "A" and "B." Results and Discussion: The approach should be carried out in the following succession: (1) To obtain samples of TDL of immature rabbits and TDL of mature rabbits (groups 1- "A" and 2- "B" accordingly) by special glass micropipette (Patent № 1495076, Russia), (2) to make lymph plasma from the samples of TDL of rabbits of group 1 and group 2, (3) to find a qualitative and quantitative components composition of lymph plasma and TDL cells of group 1 and group 2, and (4) to find the difference $(\mathrm{MF} / \Delta)$ between the lymph plasma of the components composition of groups 1 and 2. Consequently, the difference $(\Delta)$ will be the sought-for MF. To study MF proteomic analysis can be used.

Conclusions: The aging-related approach can be used to study different features of TDL, particularly cells division, identification of lymph MF, that can play a key role in access to cell dividing mechanism, and it is the way to elaborate new cancer drugs.

Key words: Aging-related approach, mitosis, lymph

\section{INTRODUCTION}

It is known that mitosis is a fundamental process and it has been researched in normal as well as in pathology. Uncontrolled mitosis is an important property of all tumors and control of mitoses is topical for cancer therapy. Aging is a biological process characterized by the progressive deterioration of physiological functions known to be the main risk factor for chronic diseases and declining health. It is proposed that there is a mutual causality between aging and aneuploidy, and it suggests modulation of mitotic fidelity as a potential means to ameliorate healthy lifespan. ${ }^{[1]}$ Univariate Cox regression analyses showed

\section{${ }^{\star}$ Corresponding author:}

Email: alexapex@yandex.ru

http://dx.doi.org/10.20530/IJIMHS425

ISSN 2056-9866 @ 2017 that a large tumor, undergoing esophagectomy, and a high mitotic rate were associated with poor survival or recurrence control. ${ }^{[2]} \mathrm{A}$ colossal amount of basic research over the past few decades has provided unprecedented insights into the highly complex process of cell division. There is an ever-expanding catalog of proteins that orchestrate, participate and coordinate in the exquisite processes of spindle formation, chromosome dynamics and the formation and regulation of kinetochore-microtubule attachments. Use of classical microtubule poisons has still been widely and often successfully used to combat a variety of cancers, but their non-selective interference in other crucial physiologic processes necessitate the identification of novel druggable components specific to the cell cycle/division pathway. ${ }^{[3]}$ Counting mitotic figures (MFs) are used in the World Health Organization grading of brain tumors. ${ }^{[4]}$ To study cells proliferation and differentiation are used research of complex combinations of multicomponent schemes. ${ }^{[5]}$ Recently, it was pointed 
out that efficient approach to the clear understanding of mitosis mechanisms is still underway. ${ }^{[6,7]}$ Regulating machine of mitoses is investigated in normal ${ }^{[8]}$ and pathology, tumors. ${ }^{[9,10]}$ At present mitosis has remained the most important prognostic factor. ${ }^{[2,11]}$ Furthermore, the increase of mitotic index in peripheral lymphoid organs is due to the presence of lymphocytopoietic factor (LF) in body fluid, particularly in the lymph. ${ }^{[12-14]}$ Mitoses can be in all tissues especially in thoracic duct central lymph because the factor is solute. In addition, it is known that lymphatic drainage plays more important roles in regulating humoral immunity and peripheral tolerance than in effector $\mathrm{T}$ cell immunity. ${ }^{[15]}$ A thoracic duct is the main part of lymphatic drainage. Some investigators arose a question whether tissue fluid/lymph, which is natural humoral environment for dermal cells, contains cytokine (s) specifically regulating cultured keratinocytes proliferation and suggested that there may be other (so far undetected) specific cytokines or that the proliferation and differentiation of keratinocytes is an effect of the combined action of all investigated cytokines. $^{[14]}$ Thoracic duct lymph (TDL) is natural humoral environment for its immunity cells. In the past century, some researchers speculated that the LF may be in TDL. The LF can control mitoses LF was shown in TDL of syngeneic rats. ${ }^{[12]}$ The same result was shown by ligature of the cervical lymph duct in guinea pigs. This was the result of dilatation of the lymphatic draining of the thymus and reduction of mitosis in the thymus. ${ }^{[16]}$ However, the definite LF quantitative/qualitative nature in TDL is still unknown. Further, LF will be called mitotic factor (MF). The aim of the work is to justify a new approach to detection of definite MF. Justification for the new approach to detection of MF composition in native TDL is based on the discovery of mitoses in TDL of normal immature rabbits, but no mitoses are detected in native TDL of normal mature rabbits. ${ }^{[17]}$ However, at present no MF has been detected neither in central lymph/TDL nor peripheral lymph and body fluid. Thoracic duct plays a key role in the drainage of lymph/body fluid. It is known that mitoses are an immunity process and mitosis is an extremely important process in an immune response and the dependence on immunity. Hence, increase of the mitoses number of TDL relates to immunity. Some works indicating that there are differences in the innate immune system of young and adult rabbits that contribute to their distinct resistance/susceptibility to RHDV infection were made. ${ }^{[18]}$ The above-mentioned data suggest that the composition of lymph cell environment/ MF of the mature rabbit's changes in comparison with the composition of lymph cell environment of the immature rabbits. Substances of MF in TDL may be cytokines, hormones, microelements, their combination and any other substances which are still undetected, specific, or nonspecific. Taking into account the foregoing and that the key role in the drainage of fluid body belongs to thoracic duct, a chance to detect a definite structure of MF in TDL becomes obvious. It is suggested that the cause of colossal number of research of mitosis is an empirical approach to the problem. For example: Finding the optimal combination (MF) of only nine components (cytokines or any proteins) requires 181440 steps. The aging-related approach to detection of an explicit mitotic factor (MF) to control mitosis through only one step is offered in the study. The aging-related approach can be used to study different features of TDL, particularly cells division, identification of lymph MF, that can play a key role in access to cell dividing mechanism, and it is the way to elaborate new cancer drugs.

\section{MATERIALS AND METHODS}

The research is carried out in two groups of Chinchilla rabbit males: Group number one - 5 immature rabbits weighing 120-240 g and group number two - 5 mature rabbits weighing 2.500-3.000 g. Native central lymph is obtained from the thoracic duct (cisterna chyli, Figure 1) of anesthetized rabbits. ${ }^{[19]}$ The thoracic duct is punctured with original glass micropipettes (Russian's patent No.1495076). Native lymph from thoracic duct can be obtained as much as needed. To realize the methodical approach a number of methods can be used. A proteomics approach is useful for identifying differentially expressed proteins in lymph plasma, and western blotting can be used for confirming the results of proteomic analysis. ${ }^{[20]}$

\section{RESULTS AND DISCUSSION}

The approach was carried out in the following succession: (1) To obtain samples of lymph from a rabbit cisterna chyli of thoracic duct (from groups 1 and 2) by glass micropipette (A and B, Figure 1). The glass micropipette with a sharpened tip is the same as an injection needle, (2) to make TDL plasma from the samples of TDL of rabbits of group 1 and group 2 (A and B, Figure 2), (3) to find a qualitative and quantitative substances composition of TDL

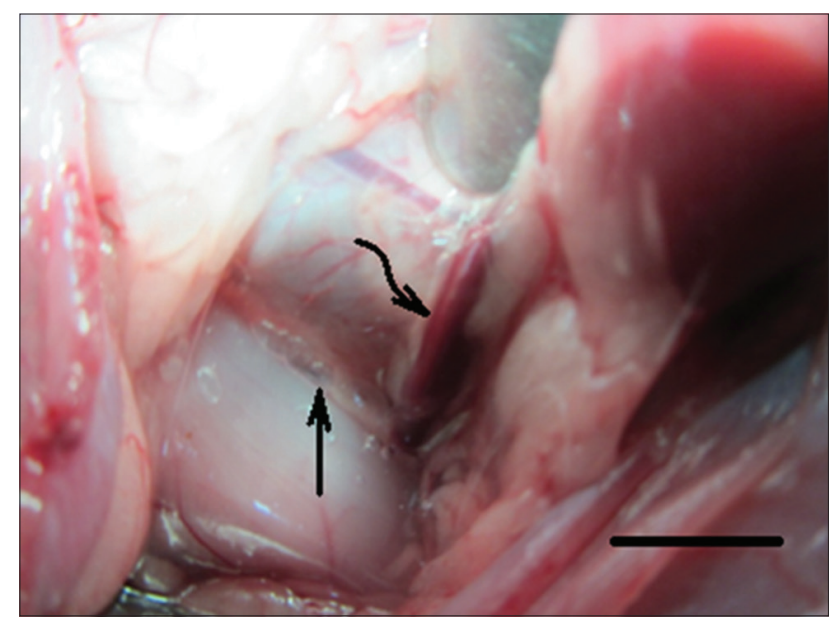

Figure 1: The cisterna chyli of thoracic duct. The straight arrows point to the cistern chyli. The winding arrow points to the left kidney vena. The scale bar is $10 \mathrm{~mm}$. 


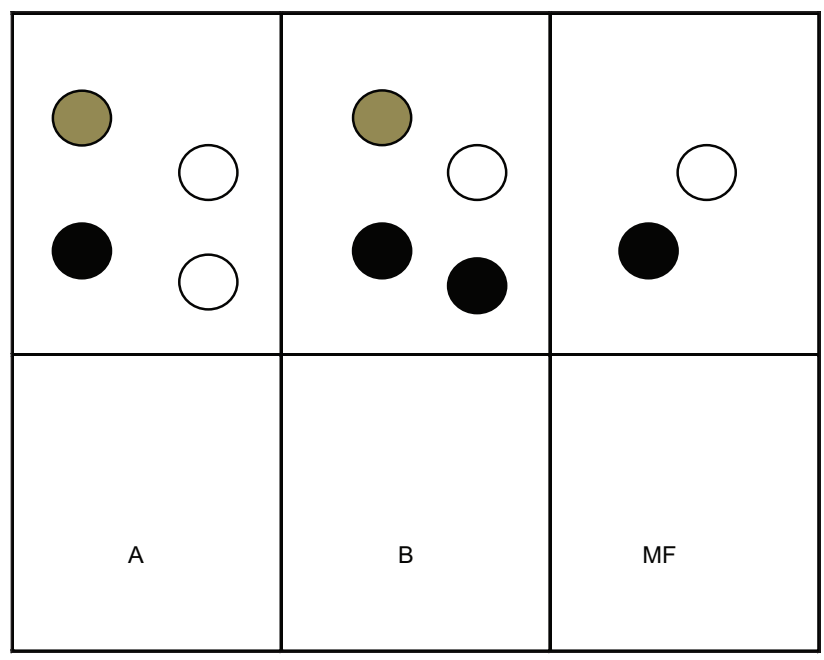

Figure 2: $A$ is the specimen of immature rabbits TDL. $B$ is the specimen of mature rabbits TDL. MF is the difference between $A$ and $B$, the explicit components combination of TDL.

- TDL components.

plasma of group 1 and group 2, and (4) to find the difference $(\Delta)$ between the TDL plasma composition of group 1 and the TDL plasma composition of group 2. Consequently, the difference $(\Delta)$ will be the sought-for MF (Figure 2). To realize the methodical approach a number of methods can be used. A proteomics approach is useful for identifying differentially expressed proteins in lymph plasma, and western blotting can be used for confirming the results of proteomic analysis. ${ }^{[20]}$ Analytical ultracentrifugation has become a widely used biomolecular research technique for determining sample purity, characterizing assembly and disassembly mechanisms of biomolecular complexes, determining subunit stoichiometries, detecting and characterizing macromolecular conformational changes, and measuring equilibrium constants and thermodynamic parameters for self- and hetero-associating systems. ${ }^{[21]}$ Application of a two-dimensional (2D) chromatographic proteomic approach, the $2 \mathrm{D}$ protein fractionation system (PF2D), can identify numerous differentially expressed proteins. ${ }^{[22]} 2 \mathrm{D}$ gel electrophoresis enables the separation of complex mixtures of proteins according to isoelectric point (pI), molecular mass (Mr), solubility, and relative abundance. ${ }^{[23]}$ Chromatography, capillary electrophoresis and mass spectrometry (MS) have become the most used proteomics methods. These techniques are also under constant development. ${ }^{[24]}$ The unprecedented increase in the number of new protein sequences arising from genomics and proteomics highlights directly the need for methods to rapidly and reliably determine the molecular and cellular functions of these proteins. One such approach, structural genomics, aims to delineate the total repertoire of protein folds, thereby providing $2 \mathrm{D}$ portraits for all proteins in a living organism and to infer molecular functions of the proteins. ${ }^{[25]}$ Regardless of the method employed, the ultimate goal of protein fractionation is to enable more protein analysis by today's current proteomics technologies, such as one-dimensional electrophoresis (1-DGE) or
2-DGE, and liquid-chromatography and tandem MS (LCMS/MS). ${ }^{[26]}$ Encouraging progress is observed in structure refinement which aims at drawing template structures closer to the native; this has been mainly driven by the use of multiple structure templates and the development of hybrid knowledge-based and physics-based force fields. For free modeling, exciting examples have been witnessed in folding small proteins to atomic resolutions. ${ }^{[27]} \mathrm{MF}$ can be a progenitor of multipurpose pharmaceutical as well as strongly peculiar pharmaceutical. In this context, it was discovered that TDL has different immunocompetent cells, including dendritic cell (DC) subsets. ${ }^{[28,29]}$ It can be suggested that some DCs mitotic figures can be observed in TDL of immature rabbits in prophase and they keep their processes. Mac Keon et al. reported that DCs play a pivotal role in the orchestration of immune responses, and are thus key targets in cancer vaccine design. Since the 2010 FDA approval of the first cancer DC-based vaccine (Sipuleucel-T), there has been a surge of interest in exploiting these cells as a therapeutic option for the treatment of tumors of diverse origin. In spite of the encouraging results obtained in the clinic, many elements of DC-based vaccination strategies need to be optimized. ${ }^{[30]}$ It can be suggested that few DCs mitotic figures can be observed in TDL of immature rabbits. Also to study MF and TDL cells, the following system of methods can be used: FACS separation of interphase and mitotic cells, including mitotic subphases, it can also be combined with proteomic analysis by mass spectrometry, specific intracellular immunolabeling protocols. ${ }^{[31]}$

Further, it is planned to assess mitoses density in the range from newborn rabbits to mature rabbits. Immature rabbits (group A) with maximum mitoses number will be used to study MF of TDL and cells of TDL.

\section{CONCLUSION}

The aging-related approach can be used as a model to study different features of TDL, particularly cells division, identification of MF that can play a key role in access to cell dividing mechanism and it is the way to elaborate new cancer drugs.

\section{ACKNOWLEDGMENT}

The author thank Galina Igorevna Kuznetsova for help in translation of the manuscript.

\section{REFERENCES}

1. Macedo JC, Vaz S, Logarinho E. Mitotic dysfunction associated with aging hallmarks. Adv Exp Med Biol 2017;1002:153-88. Available from: https://www.link. springer.com/chapter/10.1007\%2F978-3-319-57127-0_7. doi: 10.1007/978-3-319-57127 0_7. 
2. Pence K, Correa AM, Chan E, Khaitan P, Hofstetter W, Kim MP. Management of esophageal gastrointestinal stromal tumor: Review of one hundred seven patients. Dis Esophagus 2017;30:1-5. Available from: https://www.academic.oup. com/dote/article-abstract/30/12/1/4096636?redirectedFrom =fulltext. doi: $10.1093 /$ dote $/$ dox064 .

3. Agarwal S, Varma D. Targeting mitotic pathways for endocrine-related cancer therapeutics. Endocr Relat Cancer 2017;24:T65-82. Available from: http://www. erc.endocrinology-journals.org/content/24/9/T65.long. doi: 10.1530/ERC-17-0080.

4. Elmaci İ, Altinoz MA, Sari R, Bolukbasi FH. Phosphorylated histone $\mathrm{H} 3$ (PHH3) as a novel cell proliferation marker and prognosticator for meningeal tumors: A Short review. Appl Immunohistochem Mol Morphol 2017. Available from: https://www.insights.ovid.com/pubmed?pmid=28777144. doi: 10.1097/PAI.0000000000000499.

5. Yin $\mathrm{X}, \mathrm{Xu} \mathrm{X}$, Zhao Y, Wang ZJ, Wang HY, Hu ZB, et al. Comparison of several optimization schemes for the induction and expansion of antibody-mediated high efficiency CIK (AMHE-CIK) in vitro. Zhongguo Shi Yan Xue Ye Xue Za Zhi 2016;24:191-6.

6. Cross FR, Umen JG. The chlamydomonas cell cycle. Plant J 2015;82:370-92.

7. Kitagawa M, Lee SH. The chromosomal passenger complex (CPC) as a key orchestrator of orderly mitotic exit and cytokinesis. Front Cell Dev Biol 2015;3:14.

8. Chang L, Barford D. Insights into the anaphase-promoting complex: A molecular machine that regulates mitosis. Curr Opin Struct Biol 2014;29:1-9.

9. Oku Y, Tareyanagi C, Takaya S, Osaka S, Ujiie H, Yoshida K, et al. Multimodal effects of small molecule ROCK and LIMK inhibitors on mitosis, and their implication as anti-leukemia agents. PLoS One 2014;9:e92402.

10. Lampaki S, Lazaridis G, Zarogoulidis K, Kioumis I, Papaiwannou A, Tsirgogianni K, et al. Defining the role of tyrosine kinase inhibitors in early stage non-small cell lung cancer. J Cancer 2015;6:568-74. Available from: http:// www.jcancer.org/v06p0568.htm. doi: 10.7150/jca.11893. eCollection 2015.

11. Pathol A, van Diest PJ, van der Wall E, Baak JP. Prognostic value of proliferation in invasive breast cancer: A review. J Clin Pathol 2004;57:675-81.

12. Yamashita A, Fukumoto T, Miyamoto M. Studies on lymph humoral factor. Evidence for a lymphocytopoietic factor in rat thoracic duct lymph. Immunology 1976;30:349-59.

13. Yamashita A, Fukumoto T, Miyamoto M. Studies on lymph humoral factor. Biological characteristics of a lymphocytopoietic factor in rat thoracic duct lymph. Immunology 1977;32:651-6.

14. Domaszewska-Szostek A, Zaleska M, Olszewski WL. The influence of tissue fluid/lymph cytokines and growth factors on human keratinocyte proliferation and differentiation. Transplant Proc 2009;41:3269-71.

15. Thomas SN, Rutkowski JM, Pasquier M, Kuan EL, Alitalo K, Randolph GJ, et al. Impaired humoral immunity and tolerance in K14-VEGFR-3-ig mice that lack dermal lymphatic drainage. J Immunol 2012;189:2181-90. Available from: http://www.jimmunol.org/content/189/5/2181.long. doi: 10.4049/jimmunol.1103545.
16. Kotani M. Inhibition of mitosis in the guinea pig thymus after ligation of draining efferent lymphatics. Lymphology 1998;31:21-6.

17. Kuznetsov AV. Mitoses in thoracic duct lymph of rabbits. Sci Agri 2015;9:89-92. Available from: http//www.pscipub.com/ SA. doi: 10.15192/PSCP.SA.2015.9.2.8992.

18. Marques RM, Costa-E-Silva A, Águas AP, Teixeira L, Ferreira PG. Early inflammatory response of young rabbits attending natural resistance to calicivirus (RHDV) infection. Vet Immunol Immunopathol 2012;150:181-8.

19. Kuznetsov AV. A new procedure for sampling lymph in animals. J Exp Biol Med 1993;116:329-31.

20. Wu Z, Xia R, Yin X, Huo Y, Zhu G, Wu S, et al. Proteomic analysis of duodenal tissue from escherichia coli F18resistant and -susceptible weaned piglets. PLoS One 2015;10:e0127164. Available from: http://www.journals.plos. org/plosone/article?id=10.1371/journal.pone.0127164. doi: 10.1371/journal.pone.0127164.

21. Cole JL, Hansen JC. Analytical ultracentrifugation as a contemporary biomolecular research tool. J Biomol Tech 1999;10:163-76.

22. Wang L, Yang W, Ju W, Wang P, Zhao X, Jenkins EC, et al. A proteomic study of hutchinson-gilford progeria syndrome: Application of 2D-chromotography in a premature aging disease. Biochem Biophys Res Commun 2012;417:1119-26.

23. Görg A, Weiss W, Dunn MJ. Current two-dimensional electrophoresis technology for proteomics. Proteomics 2004;4:3665-85. Available from: http://www.onlinelibrary. wiley.com/doi/10.1002/pmic.200401031. doi/10.1002/ pmic.200401031.

24. Conrotto P, Souchelnytskyi S. Proteomic approaches in biological and medical sciences: Principles and applications. Exp Oncol 2008;30:171-80.

25. Zhang C, Kim SH. Overview of structural genomics: From structure to function. Curr Opin Chem Biol 2003;7:28-32.

26. Hey J, Posch A, Cohen A, Liu N, Harbers A. Fractionation of complex protein mixtures by liquid-phase isoelectric focusing. Methods Mol Biol 2008;424:225-39. Available from: https://www.link. springer.com/protocol/10.1007\%2F978-1-60327-064-9_19. doi: 10.1007/978-1-60327-0649_19.

27. Zhang Y. Progress and challenges in protein structure prediction. Curr Opin Struct Biol 2008;18:342-8.

28. Kuznetsov AV. Phenotypes of dendritic cells in central lymph of healthy rabbits and during correction of experimental atherosclerosis. Biull Eksp Biol Med 1992;114:241-2.

29. Milling S, MacPherson G. Isolation of rat intestinal lymph DC. Methods Mol Biol 2010;595:281-97. Available from: https://www.link.springer.com/protocol/ 10.1007\%2F978-1-60761-421-0_19. doi: 10.1007/978-160761-421-0_19.

30. Mac Keon S, Ruiz MS, Gazzaniga S, Wainstok R. Dendritic cell-based vaccination in cancer: Therapeutic implications emerging from murine models. Front Immunol 2015;6:243

31. Ly T, Whigham A, Clarke R, Brenes-Murillo AJ, Estes B, Madhessian D, et al. Proteomic analysis of cell cycle progression in asynchronous cultures, including mitotic subphases, using PRIMMUS. Elife 2017;6:e27574. 